\title{
Rastreo de abuso de alcohol: comparación de distintos cuestionarios
}

Screening for Problem Drinking.

Comparison of CAGE and AUDIT. Bradley KA, Bush KR, Mc Donell MB et al. J Gen Intern Med 1998;13:379-88

\begin{abstract}
Objetivo
Comparar las características de tres custionarios autoadministrados para detectar abuso y uso excesivo de alcohol. Los cuestionarios evaluados fueron el CAGE, el AUDIT (test de identificación de problemas por uso de alcohol), y el CAGE 'aumentado'.
\end{abstract}

\section{Diseño}

Estudio de corte transversal.

\section{Lugar}

Tres clínicas generales de veteranos, EE.UU.

\section{Pacientes}

Se tomó una muestra aleatorizada de un grupo de pacientes ambulatorios de sexo masculino, que hubieran consumido al menos cinco tragos en el año previo (bebedores). En el muestreo se sobrerepresentaron a los bebedores excesivos.

\section{Descripción de los tests en estudio}

Se utilizaron tres instrumentos (ver apéndice): CAGE ( 4 items), AUDIT (10 items), y el 'CAGE Aumentado' (7 items).

\section{Descripción de test de referencia}

Se realizó una entrevista telefónica con la encuesta de consumo de alcohol de la Organización Mundial de la Salud. Esta interroga detalladamente a los pacientes sobre el tipo y la cantidad de bebida consumida en días típicos de bajo, medio y alto consumo. También se diagnosticó abuso o dependencia del alcohol según criterios del DSM-III-R (del in- glés Diagnostics Statistics Mental disorders, tercera edición revisada). A la mitad de los entrevistados se los llamó antes de la encuesta postal, y a la otra mitad se los llamó después, para explorar la influencia de la entrevista previa en el cuestionario postal. Los entrevistadores estaban ciegos a las respuestas de la encuesta postal.

\section{Medición de reultados principales}

Según las entrevistas se clasificaron a los pacientes en cuatro categorías diagnósticas: 1 ) bebedores excesivos: los que tomaban más de 14 tragos por semana en un mes típico, o a los que bebían 50 más tragos en un mismo día al menos una vez al mes, 2) abuso o dependencia en a lgún momento de la vida: criterios del DSM-III-R, 3) abuso o dependencia activa (un subgrupo de los anteriores que hayan tenido sintomas en el último año) y 4) Bebedores excesivos o abuso o dependencia activos en el último año. Se utilizaron curvas ROC* para comparar los distintos tests con el test de referencia

\section{Resultados Principales}

De 393 pacientes elegibles, 261 (66\%) respondieron la encuesta postal y telefónica. El 2.2\% cumplía criterios de abuso o dependencia en algún momento de la vida, el 34\% de bebedores excesivos, y el $40 \%$ de bebedores excesivos y/o abuso o dependencia activos. EL CAGE aumentado fue mejor para detectar abuso a dependencia activo o en algún momento de la vida ( $p<0.001$ comparado con el CAGE o el AUDIT). EL AUDIT fue superior para detectar bebedores excesivos $(p<0.001)$. Para identificar bebedores excesivos y abuso o dependencia activa el AUDIT fue también superior a los otros dos $(p<0.001)$. Las áreas bajo la curva oscilaron entre 0.654 y 0.871.

\section{Características operativas de los distintos tests}

\begin{tabular}{|c|c|c|c|c|c|c|c|c|c|}
\hline \multirow[t]{2}{*}{ Test } & \multirow[t]{2}{*}{$\begin{array}{l}\text { Criterio de } \\
\text { Positividad }\end{array}$} & \multicolumn{2}{|c|}{$\begin{array}{c}\text { Abuso Activo } \\
\text { o Dependencia Activo } \\
(n=56)\end{array}$} & \multicolumn{2}{|c|}{$\begin{array}{l}\text { Abuso Activo o } \\
\text { Dependencia en algún } \\
\text { momento }(n=127)\end{array}$} & \multicolumn{2}{|c|}{$\begin{array}{l}\text { Bebedores excesivos } \\
\qquad(\mathrm{n}=89)\end{array}$} & \multicolumn{2}{|c|}{$\begin{array}{l}\text { Bebedor Excesivo y/o } \\
\text { Abuso o Dependencia } \\
\text { Activa }(n=105)\end{array}$} \\
\hline & & $\begin{array}{c}\text { Sens-Espec } \\
\%\end{array}$ & $\begin{array}{l}\text { Coef de } \\
\text { Prob+ }\end{array}$ & $\begin{array}{c}\text { Sens-Espec } \\
\%\end{array}$ & $\begin{array}{l}\text { Coef de } \\
\text { Prob+ }\end{array}$ & $\begin{array}{c}\text { Sens-Espec } \\
\%\end{array}$ & $\begin{array}{l}\text { Coef de } \\
\text { Prob+ }\end{array}$ & $\begin{array}{c}\text { Sens-Espec } \\
\%\end{array}$ & $\begin{array}{l}\text { Coef de } \\
\text { Prob+ }\end{array}$ \\
\hline \multirow{3}{*}{ CAGE } & $\geq 1$ & $89-53$ & 1.9 & $72-59$ & 1.8 & $74-53$ & 1.6 & $77-58$ & 1.9 \\
\hline & $\geq 2$ & $77-79$ & 3.7 & $53-86$ & 3.7 & $49-75$ & 2.0 & $53-81$ & 2.8 \\
\hline & $\geq 3$ & $50-93$ & 7.3 & $30-97$ & 10.0 & $29-91$ & 3.1 & $29-92$ & 3.7 \\
\hline \multicolumn{10}{|l|}{ CAGE } \\
\hline \multirow[t]{4}{*}{ Aumentado } & $\geq 1$ & $98-46$ & 1.8 & $82-54$ & 1.8 & $85-49$ & 1.7 & $87-53$ & 1.8 \\
\hline & $\geq 2$ & $91-68$ & 2.8 & $65-74$ & 2.5 & $70-68$ & 2.2 & $72-74$ & 2.8 \\
\hline & $\geq 3$ & $73-83$ & 4.4 & $47-89$ & 4.2 & 44 & 2.1 & $49-85$ & 3.2 \\
\hline & $\geq 4$ & $55-93$ & 7.6 & $33-97$ & 11.1 & 79 & 3.9 & $34-94$ & 5.4 \\
\hline \multirow[t]{3}{*}{ AUDIT } & $\geq 4$ & $79-54$ & 1.7 & $61-54$ & 1.3 & $92-67$ & 2.8 & $87-70$ & 2.9 \\
\hline & $\geq 6$ & $70-78$ & 3.2 & $49-84$ & 3.0 & $70-87$ & 5.4 & $66-90$ & 6.8 \\
\hline & $\geq 8$ & $66-86$ & 4.8 & $39-89$ & 3.5 & $57-92$ & 6.9 & $55-96$ & 12.3 \\
\hline
\end{tabular}

\section{Conclusiones}

En esta población masculina en atención primaria, el CAGE aumentado fue el mejor test para detectar abuso o dependencia activa. Para identificar a bebedores excesivos y/o abuso o dependencia en el último año, el AUDIT fue el más certero.

\section{COMENTARIO}

Los problemas relacionados al alcohol son muy prevalentes. Entre el 10 y $36 \%$ de los pacientes vistos en atención primaria padecen abuso o dependencia al alcohol ${ }^{1}$. Estos problemas generan mucha morbimortalidad, y son muy difíciles de diagnosticar. No existe ningún test de laboratorio que sirva para rastrear problemas relacionados al alco$\mathrm{hol}^{2}$. Aunque la gamma glutamil transpeptidasa es el test bioquímico más sensible, sólo logra detectar al 25 a $36 \%$ de los abusadores.
Todas Las agrupaciones preventivas recomiendan rastrear el consumo de alcohol en todos los pacientes desde la adolescencia por medio de cuestionarios estructurados ${ }^{2}$. De los varios instrumentos desarrollados para evaluar hábitos de consumo de alcohol ${ }^{3}$, el CAGE y el AUDIT son de los más estudiados. La ventaja del CAGE es que es corto y fácil de recordar. Evalúa los problemas relacionados al alcohol sufridos alguna vez, pero no distingue entre un problema activo o uno muy dis- 
tante en el pasado. Tampoco es muy bueno para detectar a bebedores excesivos. El 'CAGE aumentado' intenta mejorar estos defectos sin alargar demasiado el cuestionario. El AUDIT es un test más extenso, diseñado específicamente para detectar a bebedores excesivos y problemas relacionados con el alcohol en el año previo. Los autores utilizaron el método de curvas ROC* para comparar los distintos tests. Este método evalua la capacidad de los distintos tests para discriminar entre enfermos y sanos. Cuanto más cerca de 1 es el área bajo la curva ROC* mayor es la capacidad de discriminación. En esta población de hombres en un ámbito de atención primaria el CAGE aumentado fue el más útil para detactar tanto problemas en el pasado sino también abuso o dependencia actual. El AUDIT resultó superior para la detección de bebedores excesivos y personas con abuso o dependencia en el último año. La sensibilidad de los tests fue menor a la reportada en otros estudios. Esto probablemente se deba a que los cuestionarios fueron autoadministrados por los pacientes, mientras que en otros estudios se los administraba algún profesional de la salud. Otro problema de este tipo de estudios que evalúan encuestas postales es el sesgo de respuesta. Como en este estudio ellos mandaban los cuestionarios junto con distintas preguntas sobre hábitos generales, pudieron evaluar este tipo de sesgo. Hallaron que los pacientes que no respondían los cuestionarios específicos de alcohol y los que se negaron a la entrevis- ta diagnóstica tenían más prevalencia de consumo excesivo.

La elección del instrumento para rastreo depende del objetivo del mismo. El CAGE aumentado fue siempre mejor que el CAGE y parece ser el indicado para identificar pacientes con problemas activos relacionados al alcohol (abuso o dependencia). En cambio, para la identificación de bebedores excesivos, en los cuales el médico de atención primaria puede ayudar a cambiar los hábitos, el AUDIT fue levemente superior al CAGE aumentado. Esta diferencia se debió principalmente a la pregunta 3 del AUDIT (ver apéndice), la cual podría agregarse al CAGE aumentado para aumentar la sensibilidad*, que en este caso es la característica más deseable del test. Una advertencia al respecto es que no conocemos las características operativas de estos tests en nuestro país, y es posible que no sean las mismas debido al distinto patrón cultural de la población.

Un estudio reciente que evalua distintos cuestionarios en mujeres complementa este estudio en población masculina. El mismo concluye que el CAGE tiene una sensibilidad pobre comparada con el AUDIT ${ }^{4}$. Como conducta de rutina en el consultorio, el CAGE aumentado parece ser test de rastreo de elección, y el uso del AUDIT podría reservarse si el médico lo tiene disponible o en los pacientes que necesiten una evaluación más detallada.

\section{Apéndice: Descripción de los tests para rastreo de problemas por alcohol y su codificación}

CAGE: 1 . Cut-down = disminuir: ¿Alguna vez sintió necesidad de disminuir su consumo de alcohol?

2. Annoyed = molesto: ¿Alguna vez alguien criticó su consumo de alcohol?

3. Guilty = culpable ¿Alguna vez se sintió mal o culpable en relación a la bebida?

4. Eye opener = tomar a la mañana ¿Alguna vez tomó un trago a la mañana para calmar los nervios o quitar una resaca?

Puntaje: Un punto por cada respuesta positiva. De uno a cuatro puntos. Se considera positivo 2 o más puntos.

AUDIT (del inglés Alcohol Use Disorders Identification Test, test para la identificación de problemas relacionados al alcohol y de consumo)

\begin{tabular}{|c|c|c|c|c|c|}
\hline Pregunta & 0 & 1 & 2 & 3 & 4 \\
\hline 1. ¿Con qué frecuencia tomó durante el último año alguna bebida que contenga alcohol? & Nunca & 1 por & $\begin{array}{l}2-4 \\
x \text { mes o } \\
\text { menos }\end{array}$ & $\begin{array}{l}2-3 \\
x \text { mes }\end{array}$ & $\begin{array}{l}\geq 4 \\
x \text { semana }\end{array}$ \\
\hline 2.¿Cuántas copas de una bebida alcohólica tomó en un típico día en el que tomó durante el último año? & Ninguna & $1-2$ & $3-4$ & $5-6$ & $7-9^{*}$ \\
\hline 3.¿Cuántas veces en el último año tomó seis o más copas en una misma ocasión? & Nunca & $\begin{array}{l}\text { Menos } \\
\text { que } 1 \\
\text { por mes }\end{array}$ & $\begin{array}{l}1 \text { por } \\
\text { pormes }\end{array}$ & $\begin{array}{l}1 \text { por } \\
\text { semana }\end{array}$ & $\begin{array}{l}\text { Diario o } \\
\text { casi } \\
\text { diario }\end{array}$ \\
\hline 5.¿Cuántas veces notó en el último año que no pudo hacer lo que se esperaba de usted debido al alcohol? & Nunca & $\begin{array}{l}\text { Menos } \\
\text { que } 1 \\
\text { pormes }\end{array}$ & $\begin{array}{l}1 \text { por } \\
\text { mes }\end{array}$ & $\begin{array}{l}1 \text { por } \\
\text { semana }\end{array}$ & $\begin{array}{l}\text { Diario o } \\
\text { casi } \\
\text { diario }\end{array}$ \\
\hline $\begin{array}{l}\text { 6. ¿Cuántas veces notó en el último año que necesitaba una copa a la mañana para empezar el día luego de } \\
\text { beber mucho la noche prvia? }\end{array}$ & Nunca & $\begin{array}{l}\text { Menos } \\
\text { que } 1 \\
\text { pormes }\end{array}$ & $\begin{array}{l}1 \text { por } \\
\text { mes }\end{array}$ & $\begin{array}{l}1 \text { por } \\
\text { semana }\end{array}$ & $\begin{array}{l}\text { Diario o } \\
\text { casi } \\
\text { diario }\end{array}$ \\
\hline 7. ¿Cuántas veces sintió culpa o remordimientos en el último año luego de tomar? & Nunca & $\begin{array}{l}\text { Menos } \\
\text { que } 1 \\
\text { por mes }\end{array}$ & $\begin{array}{l}1 \text { por } \\
\text { mes }\end{array}$ & $\begin{array}{l}1 \text { por } \\
\text { semana }\end{array}$ & $\begin{array}{l}\text { Diario o } \\
\text { casi } \\
\text { diario }\end{array}$ \\
\hline 8. ¿Cuántas veces en el último año le costó recordar lo que había pasado la noche previa debido al alcohol? & Nunca & $\begin{array}{l}\text { Menos } \\
\text { que } 1 \\
\text { por mes }\end{array}$ & $\begin{array}{l}1 \text { por } \\
\text { mes }\end{array}$ & $\begin{array}{l}1 \text { por } \\
\text { semana }\end{array}$ & $\begin{array}{l}\text { Diario o } \\
\text { casi } \\
\text { diario }\end{array}$ \\
\hline 9. ¿Alguna vez ha sido lastimado Ud. o alguna persona como resultado de su hábito de beber? & Nunca & \multicolumn{2}{|c|}{$\begin{array}{l}\text { Sí, pero no en } \\
\text { el último año } \\
\text { ( } 2 \text { puntos) }\end{array}$} & \multicolumn{2}{|c|}{$\begin{array}{l}\text { Sí, en el último año } \\
\text { ( } 4 \text { puntos) }\end{array}$} \\
\hline $\begin{array}{l}\text { 10. ¿Alguna vez se preocupó un pariente, amigo, un doctor o alguien del equipo de salud en relación } \\
\text { a su manera de beber o le sugirieron que disminuyera la cantidad? }\end{array}$ & Nunca & \multicolumn{2}{|c|}{$\begin{array}{l}\text { Sí, pero no en } \\
\text { el último año } \\
\text { ( } 2 \text { puntos) }\end{array}$} & \multicolumn{2}{|c|}{$\begin{array}{l}\text { Sí, en el último año } \\
\text { ( } 4 \text { puntos) }\end{array}$} \\
\hline
\end{tabular}

Un puntaje de 8 o más sugiere problemas debido al alcohol. Estos pacientes deben evaluarse en más profundidad. (1)

CAGE Aumentado: Las preguntas del CAGE + las 1 y 2 del AUDI + ¿Tuvo alguna vez problemas con el alcohol?

*Ver Glosario

Puntaje: Puntos del CAGE + 1 punto si historia de problema por consumo de alcohol +1 punto si bebedor excesivo.

Dr. Federico Augustovski

Unidad de Medicina Familiar y Preventiva. Hospital Italiano de Buenos Aires.

Referencias

1. Buchsbaum DG, Buchanan RG, Centor RM et al. Screening for alcohol abuse using CAGE scores and likelihood ratios. Ann Intern Med 1991;115:774-7

2. Put Prevention into Pratcice.Clinician's Handbook of Preventive Services. 2nd Edition. International Medical Publishers 1998. Alcohol and other drug abuse, págs 370-380.

3. http://www.sgim.org.meetings/am19/ws/subabuse.html Abstracts - July 8, 1998

4. Bradley KA, Wickizer JB, Powell SH et al. Alcohol Screening Questionnaires in Women A Critical Review. JAMA. 1998;280:166-171 